\title{
Graviton mass bounds from an analysis of bright star trajecto- ries at the Galactic Center
}

\author{
Alexander Zakharov ${ }^{1,2,3,4, \star}$, Predrag Jovanovićc ${ }^{, \star \star}$, Dusko Borka ${ }^{6, \star \star \star}$, and Vesna Borka

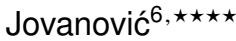 \\ ${ }^{1}$ Institute of Theoretical and Experimental Physics, 117259 Moscow, Russia \\ ${ }^{2}$ Bogoliubov Laboratory for Theoretical Physics, JINR, 141980 Dubna, Russia \\ ${ }^{3}$ National Research Nuclear University MEPhl (Moscow Engineering Physics Institute), 115409, Moscow, \\ Russia \\ ${ }^{4}$ North Carolina Central University, Durham, NC 27707, USA \\ ${ }^{5}$ Astronomical Observatory of Belgrade, Volgina 7, 11060 Belgrade, Serbia \\ ${ }^{6}$ Atomic Physics Laboratory (040), Vinča Institute of Nuclear Sciences, University of Belgrade, P.O. Box 522, \\ 11001 Belgrade, Serbia
}

\begin{abstract}
In February 2016 the LIGO \& VIRGO collaboration reported the discovery of gravitational waves in merging black holes, therefore, the team confirmed GR predictions about an existence of black holes and gravitational waves in the strong gravitational field limit. Moreover, in their papers the joint LIGO \& VIRGO team presented an upper limit on graviton mass such as $m_{g}<1.2 \times 10^{-22} \mathrm{eV}$ (Abbott et al. 2016). So, the authors concluded that their observational data do not show any violation of classical general relativity. We show that an analysis of bright star trajectories could constrain graviton mass with a comparable accuracy with accuracies reached with gravitational wave interferometers and the estimate is consistent with the one obtained by the LIGO \& VIRGO collaboration. This analysis gives an opportunity to treat observations of bright stars near the Galactic Center as a useful tool to obtain constraints on the fundamental gravity law such as modifications of the Newton gravity law in a weak field approximation. In that way, based on a potential reconstruction at the Galactic Center we obtain bounds on a graviton mass.
\end{abstract}

\section{Introduction. Centurial triumph of General Relativity}

General relativity (GR) has been discovered in November 1915 in [1, 2] as it is well-known in the literature (see, [3-7] for reference). Gravitational field is described by the following expression

$$
R_{\mu \nu}-\frac{1}{2} R g_{\mu \nu}=\frac{8 \pi G}{c^{4}} T_{\mu \nu}
$$

where $R_{\mu \nu}$ is the Ricci tensor, $g_{\mu \nu}$ is the metric tensor, $R$ is the scalar curvature, $T_{\mu \nu}$ is the stress-energy tensor, $G$ and $c$ are the Newton constant and the speed of light, respectively.

\footnotetext{
$\star$ e-mail: zakharov@itep.ru

$\star \star$ e-mail: pjovanovic@aob.rs

$\star \star \star$ e-mail: dusborka@vin.bg.ac.rs

$\star \star \star \star$ e-mail: vborka@vinca.rs
} 


\subsection{Deflection of light and Mercury anomaly}

In November 1915 (before the discovery of GR) in paper [8] A. Einstein calculated deflection of light which is double in respect to the Newtonian one (therefore, measurements of light deflection provide an effective test of a new theory of gravity) and explained the Mercury orbit anomaly found by U.J.J. Le Verrier in 1859 [9] (Le Verrier suggested also a way to resolve any anomaly, see [10] for a detailed discussion). At this period (before November 25, 1915) A. Einstein knew correct gravitational equations in vacuum

$$
R_{\mu v}=0
$$

and used them in his derivations of light deflection and the Mercury anomaly. So, Einstein derived an expression for light deflection

$$
\Theta=\frac{4 G M}{c^{2} p},
$$

where $M$ is a mass of gravitating body and $p$ is an impact parameter. In the case, if we observe light deflection near a Solar disk during solar eclipse $M=M_{\odot}$ and $p=R_{\odot}$ and $\Theta=1.75$ ". The Einstein's prediction about the light deflection has been confirmed by British astronomers on 29 May, 1919 during solar eclipse in Sobral (Brazil) and island Principe (near Africa) [11] (see, also [12, 13]). Observations at Principe confirmed GR predictions while Sobral's observations showed $\Theta=0.93$ ", but astronomers decided that these observations are not reliable. After those observations mass media were very excited because a new (and more correct) theory of gravity has been found [13]. However, during solar eclipse on May 29, 1929 in Sumatra, E. Freundlich ${ }^{1}$ obtained the light deflection angle $\Theta=2.24 \pm 0.1$ " [18]. On December 11, 1931 Freundlich reported his results to the Royal Astronomical Society, he claimed that 1) a deflection exists; 2) it is not Newton's; 3) it seems to be greater than Einstein's, after that A. Eddington claimed that "I find it difficult to believe 1.75" can be wrong" [19]. Now the Einstein's expression (3) has been checked with a very high precision [20-22] and the post-Newtonian parameter $\gamma$ is very close to 1 [23].

As it was mentioned earlier A. Einstein explained perihelion precession for Mercury [8]

$$
\phi=\frac{24 \pi^{3} L^{2}}{T^{2} c^{2}\left(1-e^{2}\right)},
$$

where $L$ and $T$ are semi-major axis and orbital period, respectively. The phenomenon is called the relativistic advance and it is extremely useful tool to evaluate parameters of the black hole, a stellar cluster and dark matter bulk distribution at the Galactic Center as we will discuss below.

\subsection{Gravitational lensing}

Gravitational lensing is based on phenomenon of light deflection in gravitational field and the theory started since papers by O. Chwolson [24], Einstein [25] and F. Zwicky [26] and now there are a number of papers describing different aspects of the phenomenon see [15, 16, 27-29] and references therein. Gravitational lens mapping is a specific case of Lagrange mapping and therefore, singularities

\footnotetext{
${ }^{1}$ In 1911 A. Einstein calculated a deflection of light in gravitational field $\Theta=\frac{2 G M}{c^{2} p}$ [14], which coincides with Newtonian one and in 1914 Einstein suggested his assistant E. Freundlich to check the result during Solar eclipse in Russian Crimea (near Feodosia) in August 1914. However, the observations were not done, Freundlich and his colleagues were taken as prisoner of war since they were German army reservists $[15,16]$, while American team led by W. W. Campbell (Lick Observatory) got rights to observe stars during the eclipse, however, they failed because there were clouds during the eclipse [17].
} 
of the mapping play a key role because the highest amplification is in a region near singularities (caustics). All singularities of the Lagrange mappings up to four dimensions are classified by V. I. Arnold [30]. Singularities with the lowest co-dimensions are folds and caustics. Asymptotic behavior of magnification near folds is given in [15] and near cusps in [31]. If masses of lenses are around solar masses and sources are located in our Galaxy or in nearby galaxies, then angular distance between images are around a few angular milli-arcseconds and it is very hard to re-solve multiple images but it will be possible to observe a amplification of background stars as it was noted in [32,33]. One can find a discussion of results of observations and their theoretical interpretation in reviews [34-38]. As it was noted in [39], microlensing is a very efficient tool to find low mass exoplanets near the habitable zone [40]. In observations of stars in Andromeda galaxy from the Northern hemisphere it is very hard to re-solve separate stars and to find manifestations of microlensing for them but it is possible to detect signature of so-called pixel lensing (or an amplification of a pixel). Such observations are described in a number of papers, see for instance [41]. Analyzing data observed by POINT-Agape collaboration, An et al. found anomaly [42] which was explained later as an exoplanet in the Andromeda galaxy [43]. If it is so, it will be the first exoplanet in another galaxy [44-47]. As it was noted [48-50] polarization observations for pixel lensing event play an extremely important role to clarify parameters of microlenses and sources which are giant stars for pixel lensing events (see also [51] for a review). Microlensing in X-ray band (including studies of microlensing for macrolensed objects) has been considered in [52-54].

\subsection{Gravitational redshift and time dilation}

Pound and Rebka found that a photon frequency is higher at the bottom of a tower than at the top of it $[55,56]$. Vessot et al. measured a maser frequency shift aboard of a spacecraft and the shift was in agreement with predictions of GR [57]. In spite of the fact that the phenomenon can be easily calculated in the framework of the GR, there are discussions of physical interpretation of these experiments [58-64].

\subsection{Inspiralling pulsars. An evidence of gravitational radiation}

Soon after the discovery of GR, A. Einstein concluded that gravitational waves have to be emitted in the case if quadrupole moment for is changed with time $[65,66]$. In his paper A. Eddington corrected a mistake in the expression describing gravitational waves, however, he believed that "they are not objective and (like absolute velocity) are not detectable by any conceivable experiment. There are merely sinuosities in the coordinate system and the only speed of propagation relevant to them is "the speed of thought" [67]. In 1936 A. Einstein and his young assistant N. Rosen submitted the paper under the "Do gravitational waves exist?" in The Physical Review. Based on the Einstein's letter to M. Born one can say that Einstein's answer was "No" [68]. However, a referee and on 23 July John Tate (who was the chief editor of PR) were not convinced and Tate asked Einstein to respond at remarks and criticism of a referee. On 27 July Einstein wrote to Tate [68]: "Dear Sir, We (Mr. Rosen and I) had sent you our manuscript for publication and had not authorized you to show it to specialists before it is printed. I see no reason to address the - in any case erroneous - comments of your anonymous expert. On the basis of this incident I prefer to publish the paper elsewhere. Respectfully, [signature]. P.S. Mr. Rosen, who has left for the Soviet Union, has authorized me to represent him in this matter." Tate answered that Einstein's unwilling contradicts to journal rules and he cannot accept such a paper. After that Einstein submitted the same paper in the Journal of Franklin Institute where the article has been published [69], but now conclusions in the paper were completely different, namely, authors claimed that gravitational waves do exist. As it was noted in [68], at the period between these two submissions 
in The Physical Review and the Journal of Franklin Institute Einstein tried to convince H. P. Robertson and L. Infeld (who substituted N. Rosen as Einstein's assistant) that the conclusions in the first version of the paper about an absence of gravitational waves are correct. At the time Robertson and Infeld were in Princeton and Einstein had very fruitful conversations with these two scientists and in the result of these conversations he arrived at the opposite conclusion that gravitational waves exist. As it was noted in [68], the important issue was that Robertson was the referee of the Einstein and Rosen paper submitted in The Physical Review. In spite of the claim about an existence of gravitational waves in their joint paper [69], N. Rosen was rather sceptical and he published a couple of papers (see, for instance $[70,71]$ ) where he argued that gravitational waves do not exist. Theoretical and experimental aspects of the quadrupole expression are discussed in a book [72], where one could find an interesting historical development of a current understanding the issue. The discussion about validity of the quadrupole expression reached a consensus when it was found that an evolution of Hulse - Taylor pulsar PSR 1913+16 [73-75] is nicely described by a simple Peters - Mathews expression which is a version of quadrupole formula for two inspiraling points [76, 77].

\subsection{Observational signatures of black holes}

If we speak about observable manifestations of black hole features we need models with a strong gravitational field to describe 1) a final stage of inspiraling (merging and ring down) binary black holes; and 2) shapes of shadows around black holes. Perhaps, very soon observers will need GR corrections and later a full GR approach to fit observational data for bright stars near the Galactic Center. Assuming that a radiation in a spectral line is emitted from a disk near a black hole horizon, it was found (and after that it was observed the X-ray $K_{\alpha}$-line) that an observed shape of the spectral line can be an important indicator of a strong gravitational field near a black hole, moreover, one can evaluate a black hole spin analyzing a spectral line structure $[78-80]^{2}$ (see also more recent reviews $[88,89]$ on the subject). Another phenomenon, where one really needs a strong gravitational field approach, is simulations of a shadow formation started since [90-93] (see also calculations of shadows for different cases [94-99] and recent reviews on the subject [100, 101]).

The problem is connected with attempts to resolve the smallest spot at the Galactic Center with VLBI interferometry in mm-band [102]. As it was noted earlier, observations of bright star trajectories near the Galactic Center could provide an efficient tool to evaluate a gravitational potential, in particular, analyzing these trajectories one can obtain constraints on parameters of black hole and stellar cluster [103] and on parameters of dark matter distribution [104-107].

Two groups of astronomers with VLT and Keck telescopes observe stars near the Galactic Center, see [108-110] and references therein. An analysis of S2 like star trajectories gives an opportunity to obtain stringent constraints on alternative theories of gravity, including $R^{n}$ theory which is a generalization of the classical GR and $n=1$ corresponds to GR $[111,112]$ (there are also stringent constraints from Solar system data [113]), and Yukawa gravity [114]. In the paper we will obtain a graviton mass constraint from analysis of trajectories of bright stars at the Galactic Center.

\section{Theories of gravity with massive graviton}

A massive gravity theory was suggested in M. Fierz and W. Pauli [115]. So-called, the van DamVeltman-Zakharov (vDVZ) discontinuity has been found [116-118], or the theory for $m_{g} \rightarrow 0$ is different from the theory with vanishing graviton mass. However, Vainshtein found a nonlinear solution for the Schwarzschild problem [119] providing a continuity at $m \rightarrow 0$ with the massless graviton

\footnotetext{
${ }^{2}$ Results of iron $K_{\alpha}$-line simulations in the framework of a simple model are given in [81-87].
} 
theory (the classical Einstein's theory of relativity). Boulware and Deser found another unpleasant feature of a massive gravity such a presence of ghosts (and related instabilities) and other pathologies from quantum field theory point of view [120]. In the last years, there was a significant progress to overcome these problems and build a consistent theory without such defects like Boulware - Deser ghosts and discontinuities [121-124] (a great step has been done in the paper [125] where the authors developed a ghost free massive gravity). Here, we will not discuss theoretical aspects of massive gravity theory and we will consider only observational features of such an approach.

In spite of the problems of current theoretical models of massive gravity in seventies Goldhaber and Nieto found a graviton mass constraint based on the assumption that a Compton wave length of graviton is around $\lambda_{g}=580 \mathrm{kpc}$ (it is around a typical distance between galaxies), and $m_{g}<2 \times 10^{-62} \mathrm{~g}=1.1 \times 10^{-29} \mathrm{eV}$ [126]. In the relativistic theory of gravitation (RTG), developed by Logunov and his group, a non-vanishing mass of graviton substitutes $\Lambda$-term in the conventional $\Lambda \mathrm{CDM}$ cosmological model and one could find that a Compton wave length for graviton has a cosmological value, so that $m_{g}<(1.3-3.2) \times 10^{-66} \mathrm{~g}$ (depending on a way for an evaluation of the quantity) [127-129], see also estimates obtained recently $m_{g}<5.2 \times 10^{-66}-1.2 \times 10^{-65} \mathrm{~g}$ taking into account constraints on quintessence parameters [130]. Constraints on $\lambda$ in Yukawa potential from Solar system data are given in [131] and analyzing these data, C. Will obtained a graviton mass constraint $m_{g}<7.2 \times 10^{-23} \mathrm{eV}$ at the $2 \sigma$ level [132]. Analyzing weak gravitational lensing data (gravitational potential reconstruction for galactic clusters based on image deformations of background galaxies), Choudhury et al. found that a Compton wavelength of massive graviton has to be $\lambda>100 \mathrm{Mpc}=3 \times 10^{21} \mathrm{~km}$ [133], therefore, $m_{g}<6 \times 10^{-32} \mathrm{eV}$. Finn and Sutton suggested to use binary pulsars PSR B1913+116 and PSR B1534+112 to evaluate a graviton mass and they found $m_{g}<7.6 \times 10^{-20} \mathrm{eV}$ with $90 \%$ confidence level [134]. Larson and Hiscock proposed to use future LISA data for observations of gravitational radiation from interacting white dwarf binary star systems, including helium cataclysmic variable ( $\mathrm{HeCV}$ ) systems and in this case one can expect to reach the following graviton mass bound $m_{g}<1 \times 10^{-24} \mathrm{eV}$ [135]. For a subsample of 400 close white dwarf binaries with high signal-to-noise ratio gravitational wave and optical data with magnitudes brighter

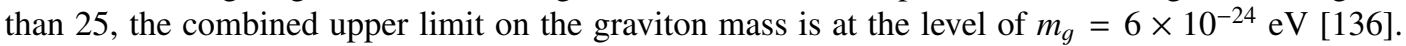
One can expect even a better estimates for ASTROD-I mission [137] because it will be possibly a next generation of space borne gravitational interferometers in space for gravitational wave detections. Many years ago, Sazhin proposed to use pulsar timing for gravitational wave detection [138] (see, also a more detailed discussion in [139]). Graviton mass constraints were obtained in [140], but later in erratum the authors noted that their approach was not correct [141], however, in paper [142] it was concluded, that one can obtain a graviton mass bound at a level $m_{g}=3 \times 10^{-22} \mathrm{eV}$, if bi-weekly observation of 60 pulsars is performed for 5 years with a pulsar rms timing accuracy of $100 \mathrm{~ns}$ and the estimate can be improved in the case if more observations for more pulsars a longer observation period would be done, moreover, such an estimate will be improved with a pulsar array technique [143]. There are other suggestions to evaluate a graviton mass, some of them are rather exotic and based on hardly verified assumptions [124]. Systematics of proposed experiments and observations is not well investigated, moreover, some weaknesses of the proposals for a graviton mass evaluation are pointed out in the review [124].

\section{Graviton mass constraint from gravitational wave signal}

If a graviton has a mass $m_{g}$, then a speed of gravitational wave propagation could differ from $c$ and we have a dispersion relation [23, 132]

$$
\frac{v_{g}^{2}}{c^{2}}=1-\frac{m_{g}^{2} c^{4}}{E^{2}}
$$


where $E$ is a graviton energy. Gravitons with different energies propagate with different velocities. Assume that we have gravitational waves and electromagnetic waves from the same source (from supernova explosion, for instance). In this case we have [23, 132]

$$
1-\frac{v_{g}}{c}=5 \times 10^{-17}\left(\frac{200 \mathrm{Mpc}}{D}\right)\left(\frac{\Delta t}{1 \mathrm{~s}}\right),
$$

where $\Delta t=\Delta t_{a}-(1+z) \Delta t_{e}$ is the time difference, where $\Delta t_{a}$ and $\Delta t_{e}$ are the differences in arrival time and emission time of the two signals, respectively, and $z$ is the redshift of the source. Usually $\Delta t_{e}$ is unknown, however, one could find an upper limit for $\Delta t_{e}$ (for instance from a theoretical model), therefore, one could evaluate $1-\frac{v_{g}}{c}$, therefore, $m_{g}$. Following $[23,132]$ and assuming that the frequency of gravitational wave is $v$ and $h v \gg m_{g} c^{2}$ ( $h$ is Planck's constant), therefore, we have $\frac{v_{g}}{c} \approx 1-\frac{1}{2} \frac{h}{\lambda_{g} v}$, where $\lambda_{g}=\frac{h}{m_{g} c}$ or $\lambda_{g} \approx \frac{1}{2} \frac{1}{\sqrt{1-v_{g} / c}}$. If one has an upper limit for $1-v_{g} / c$, it can be re-written as a lower limit for $\lambda_{g}$, as the following expression [23, 132]

$$
\lambda_{g}=3 \times 10^{12} \mathrm{~km}\left(\frac{200 \mathrm{Mpc}}{D} \frac{v}{100 \mathrm{~Hz}}\right)\left(\frac{1}{v \Delta t}\right) .
$$

It is a lucky case if one observes electromagnetic and gravitational radiation from the same source. But even in the case if only gravitational radiation has been detected as it was noted [132] because gravitational wave signal with a massive graviton will be different from signal for a graviton with a vanishing mass and in this case for $D \approx 200 \mathrm{Mpc}, v \approx 100 \mathrm{~Hz}, v \Delta t \sim \rho^{-1} \approx 0.1$ The result is $\lambda_{g}>10^{13} \mathrm{~km}$. Based on ideas expressed in [23, 132], the LIGO/VIRGO collaboration obtained the same estimate for the Compton wavelength of a massive graviton [144, 145] and detections of one additional event and one suspected case did not change the estimate [146].

\section{Graviton mass constraint from an analysis of bright star trajectories at the Galactic Center}

We use a modification of the Newtonian potential corresponding to a massive graviton case $[23,132$, 147]:

$$
V(r)=-\frac{G M}{(1+\delta) r}\left[1+\delta e^{-\left(\frac{r}{\lambda}\right)}\right],
$$

where $\delta$ is a universal constant (we put $\delta=1$ ). In our previous studies [114] we found constraints on parameters of Yukawa gravity. As it was described in $[148,149]$ we used observational data from NTT/VLT [108]. If we wish to find a limiting value for $\lambda_{x}$, so that $\lambda>\lambda_{x}$ with a probability $P=1-\alpha$ (where we select $\alpha=0.1$ ) normalized depending on $\lambda_{x}$ has to be equal to the threshold depending on degree of freedom $v$ and parameter $\alpha$ or in other words, $\chi^{2}\left(\lambda_{x}\right)=\chi_{v, \alpha}^{2}$. Computing these quantities we obtain $\lambda_{x}=2900 \mathrm{AU} \approx 4.3 \times 10^{11} \mathrm{~km}$. Now we obtain the upper limit on a graviton mass and we could claim that with a probability $P=0.9$, a graviton mass should be less than $m_{g}=2.9 \times 10^{-21} \mathrm{eV}$ ( since $m_{g}=h c / \lambda_{x}$ ) in the case of $\delta=1[148]$.

\section{Conclusions}

As it was noted earlier, our graviton mass estimate is slightly greater than estimate with LIGO interferometer, however, a) our approach has a minimal number of assumptions and our estimate was obtained 
in independent way; b) our estimate is consistent with LIGO's one; c) our estimate will be definitely improved with forthcoming facilities such as GRAVITY, E-ELT and TMT [150-152] because more precise observations of bright star orbits will give an opportunity to reconstruct a gravitational potential at the Galactic Center in a more accurate way, therefore, one can expect a better estimates for $\lambda$ parameter and a graviton mass. However, such a progress will be not very rapid because of an exponential dependence of a potential on $\lambda$.

A.F.Z. thanks the organizers of the XXIII International Baldin Seminar for their kind invitation to present a plenary talk at the seminar. A.F.Z. appreciates NSF (HRD-1345219) and NASA (NNX09AV07A) at NASA CCFASE and NSF CREST Centers (NCCU, Durham, NC, USA) for a partial support. P.J., D.B.and V.B.J. wish to acknowledge the support by the Ministry of Education, Science and Technological Development of the Republic of Serbia through the project 176003 "Gravitation and the large scale structure of the Universe". P. J. also thanks ICTP - SEENET-MTP project PRJ-09 Cosmology and Strings for a partial support.

\section{References}

[1] A. Einstein, Sitzungsber. K. Preuss. Akad. Wiss. 48, 844 (1915)

[2] D. Hilbert, Göttingen Nachr. 3, 395 (1915)

[3] J. Mehra, Einstein, Hilbert and the Theory of Gravitation (Dordrecht, Boston, 1974)

[4] J. Earman and C. Glymour, Arch. Hist. and Exact. Sci. 19, 291 (1978)

[5] V. P. Vizgin and Ya.A. Smorodinskii, Physics - Uspekhi 22, 489 (1979)

[6] V. P. Vizgin, Physics - Uspekhi 44, 1283 (2001)

[7] A. A. Logunov, M. A. Mestvirishvili and V. A. Petrov, Physics - Uspekhi 47, 607 (2004)

[8] A. Einstein, Sitzungsber. K. Preuss. Akad. Wiss. 47, 831 (1915)

[9] U. J. J. Le Verrier, R. Acad. Sci. Paris 59, 379 (1859)

[10] A. F. Zakharov et al. Space Sci. Rev. 148, 301 (2009)

[11] F. Dyson, A. S. Eddington and C. R. Davidson, Phil. Trans. Roy. Soc. A. 220, 571 (1920)

[12] S. Chandrasekhar, Notes Rec. R. Soc. Lond. 30, 249 (1976)

[13] J. Eisenstaedt, The Curious History of Relativity (Princeton University Press, Princeton, 2006) $167-195$

[14] A. Einstein, Ann. Phys. 35, 898 (1911)

[15] P. Schneider, J. Ehlers and E. E. Falco, Gravitational Lenses (Springer, Berlin, 1992)

[16] A. F. Zakharov, Gravitational Lenses and Microlenses (Yanus, Moscow, 1997) (in Russian)

[17] J. Crelinsten, Hist. Stud. Phys. Scien. 14, 1 (1983)

[18] E. Freundlich, H. V. Klüber and A. V. Brunn, Zeitschrift für Astrophysik 3, 171 (1931)

[19] H. Knox-Shaw, Meet. Roy. Astron. Soc. (December 11, 1931) The Observatory 55, 1 (1932)

[20] S. S. Shapiro et al., Phys. Rev. Lett. 92, 121101 (2004)

[21] S. B. Lambert and C. Le Poncin-Lafitte, Astron. Astrophys. 499, 331 (2009)

[22] S. B. Lambert and C. Le Poncin-Lafitte, Astron. Astrophys. 529, A70 (2011)

[23] C. Will, Living Rev. Relat. 17, 4 (2014)

[24] O. Chwolson, Astron. Nachr. 221, 329 (1924)

[25] A. Einstein, Science 84, 506 (1936)

[26] F. Zwicky, Phys. Rev. 51, 290 (1937)

[27] J. Wambsganss, Living Reviews in Relativity 1, 12 (1998)

[28] A. Petters, H. Levine and J. Wambsganss, Singularity theory and gravitational lensing (Birkhäuser, Boston, 2001) 
[29] S. Mollerach and E. Roulet, Gravitational Lensing and Microlensing (World Scientific, Singapore, 2002)

[30] V. I. Arnold, Soviet Math. Surveys 38, 87 (1983)

[31] A. F. Zakharov, Astron. Astrophys. 293, 1 (1995)

[32] A. V. Byalko, Sov. Astron. 13, 784 (1970)

[33] B. Paczynski, Astrophys. J. 304, 1 (1996)

[34] B. Paczynski, Ann. Rev. Astron. Astrophys. 34, 419 (1996)

[35] E. Roulet and S. Mollerach, Phys. Rep. 279, 67 (1997)

[36] A. F. Zakharov and M. V. Sazhin, Phys. - Usp. 41, 945, (1998)

[37] A. F. Zakharov, Publ. Astron. Observ. Belgrade 75, 27 (2003)

[38] A. F. Zakharov, Phys. Part. Nucl. 39, 1176 (2008)

[39] S. Mao and B. Paczynski, Astrophys. J. 374, L37 (1991)

[40] S. Mao, Res. Astron. Astrophys. 8, 947 (2012)

[41] S. Calchi Novati et al., Astron. Astrophys. 469, 115 (2007)

[42] J. H. An et al., Astrophys. J. 601, 845 (2004)

[43] G. Ingrosso et al., Mon. Not. R. Astron. Soc. 399, 219 (2009)

[44] A. F. Zakharov, New Astron. Rev. 53, 202 (2009)

[45] G. Ingrosso et al., Gen. Relat. Grav. 43, 1047 (2010). arXiv:1001.4342v1 [astro-ph.SR]

[46] A. F. Zakharov et al., Mem. della Soc. Astron. Ital. Suppl. 15, 114 (2010)

[47] A. F. Zakharov et al. Publ. Astron. Observ. Belgrade 92, 65 (2013)

[48] G. Ingrosso et al., Month. Not. R. Astron. Soc. 426, 1496 (2012)

[49] A. F. Zakharov et al., Adv. Space Res. 54, 1319 (2014). arXiv:1312.3468v1[astro-ph.SR]

[50] G. Ingrosso et al., Physica Scripta 89, 084001 (2014). arXiv: 1310.5866v1[astro-ph.SR]

[51] A. F. Zakharov, J. Phys.: Conf. Ser. 678, 012010 (2016)

[52] A. F. Zakharov, L. C. Popović and P. Jovanović, Astron. Astrophys. 420, 881 (2004)

[53] L. C. Popovic et al., Astrophys. J. 637, 630 (2006)

[54] P. Jovanović et al. Mon. Not. R. Astron. Soc. 386, 397 (2008)

[55] R. V. Pound and G. A. Rebka, Phys. Rev. Lett. 3, 439 (1959)

[56] R. V. Pound and G. A. Rebka, Phys. Rev. Lett. 4, 337 (1960)

[57] R. F. C. Vessot et al., Phys. Rev. Lett. 45, 2081 (1980)

[58] V. V. Okorokov, arXiv:gr-qc/9902033 (1999)

[59] V. V. Okorokov, Dokl. Phys. 46, 400 (2001)

[60] L. B. Okun, K. G. Selivanov and V. L. Telegdi, Phys. - Usp. 42, 1045 (1999)

[61] L. B. Okun, K. G. Selivanov and V. L. Telegdi, Am. J. Phys. 68, 115 (2000)

[62] L. B. Okun, Mod. Phys. Lett. A 15, 1941 (2000)

[63] L. B. Okun, Mod. Phys. Lett. A 15, 2007 (2000)

[64] L. B. Okun and K. G. Selivanov, Dokl. Phys. 47, 461 (2002)

[65] A. Einstein, Sitzungsber. K. Preuss. Akad. Wiss. 688 (1916)

[66] A. Einstein, Sitzungsber. K. Preuss. Akad. Wiss. 154 (1918)

[67] A. Eddington, Proc. R. Soc. London, Ser. A 154 (1922)

[68] D. Kennefick, Phys. Today 9, 43 (2005)

[69] A. Einstein and N. Rosen, J. Franklin Inst. 223, 43 (1937)

[70] N. Rosen, in Jubilee of Relativity Theory (Helv. Phys. Acta Suppl. IV), edited by A. Mercier and M. Kervaire (Birkhauser Verlag, Basel, 1956), 171 
[71] N. Rosen, Nuovo Cim. 19, 249 (1977)

[72] D. Kennefick, Traveling at the Speed of Thought: Einstein and the Quest for Gravitational Waves (Princeton University Press, Princeton, 2007)

[73] R. A. Hulse and J. H. Taylor, Astrophys. J. 195, L51 (1975)

[74] J. H. Taylor and J. M. Weisberg, Astrophys. J. 253, 908 (1982)

[75] J. M. Weisberg, D. J. Nice and J. H. Taylor, Astrophys. J. 722, 1030 (2010)

[76] P. C. Peters and J. Mathews, Phys. Rev. 131, 435 (1963)

[77] P. C. Peters, Phys. Rev. 136, 1224 (1964)

[78] A. C. Fabian et al., Mon. Not. R. Astron. Soc. 238, 729 (1989)

[79] L. Stella, Nature 344, 747 (1990)

[80] Y. Tanaka et al., Nature 375, 659 (1995)

[81] A. F. Zakharov and S. V. Repin, Astron. \& Astrophys. 406, 7 (2003)

[82] A. F. Zakharov and S. V. Repin, Astron. Rep. 47, 733 (2003)

[83] A. F. Zakharov and S. V. Repin, Nuovo Cim. 118B, 1193 (2003)

[84] A. F. Zakharov and S. V. Repin, Adv. Space Res. 34, 2544 (2004)

[85] A. F. Zakharov and S. V. Repin, Mem. S. A. It. della Suppl. 7, 60 (2005)

[86] A. F. Zakharov and S. V. Repin, New Astron. 11, 405 (2006)

[87] A. F. Zakharov, Phys. Atom. Nucl. 70, 159 (2007)

[88] A. C. Fabian and R. R. Ross, Space Sci. Rev. 157, 167 (2010)

[89] P. Jovanović, New Astron. Rev. 56, 37 (2012)

[90] P. Young, Phys. Rev. D 14, 3281 (1976)

[91] S. Chandrasekhar, Mathematical Theory of Black Holes (Oxford: Clarendon Press, 1983)

[92] H. Falcke, F. Melia and E. Agol, Astrophys. J. 528, L13 (2000)

[93] F. Melia and H. Falcke, Ann. Rev. Astron. Astrophys. 39, 309 (2001)

[94] A. F. Zakharov et al., Astron. \& Astrophys. 442, 795 (2005)

[95] A. F. Zakharov et al., New Astron. 10, 479 (2005). astro-ph/0411511

[96] F. De Paolis et al., Gen. Rel. Grav. 43, 977 (2010)

[97] A. F. Zakharov et al., New Astron. Rev. 56, 64 (2012)

[98] A. F. Zakharov, Phys. Rev. D 90, 062007 (2014)

[99] A. F. Zakharov, J. Astrophys. Astr. 36, 539 (2015)

[100] H. Falcke and S. Markoff, Class. Quan. Grav. 30, 244003 (2013)

[101] T. Johannsen, Class. Quan. Grav. 33, 113001 (2016)

[102] S. S. Doeleman et al., Nature 455, 78 (2008)

[103] A. A. Nucita et al., Publ. Astron. Soc. Pac. 119, 349 (2007)

[104] A. F. Zakharov et al., Phys. Rev. D 76, 062001 (2007)

[105] A. F. Zakharov et al., Space Sci. Rev. 48, 301 (2009)

[106] A. F. Zakharov et al. Phys. At. Nucl. 73 , 1870 (2010)

[107] V. I. Dokuchaev and Yu. N. Eroshenko Phys.-Usp. 58, 772 (2015)

[108] S. Gillessen et al., Astrophys. J. 707, L114 (2009)

[109] S. Gillessen et al., Nature 481, 51 (2012)

[110] L. Meyer et al., Science 338, 84 (2012)

[111] D. Borka et al., Phys. Rev. D 85, 124004 (2012)

[112] A. F. Zakharov et al. Adv. Space Res. 54, 1108 (2014)

[113] A. F. Zakharov et al., Phys. Rev. D 74, 107101 (2006) 
[114] D. Borka et al., J. Cosmol. Astropart. Phys. JCAP11 (2013) 050

[115] M. Fierz and W. Pauli, Proc. R. Soc. London, Ser. A 173, 211 (1939)

[116] V. I. Zakharov, JETP Lett. 12, 447 (1970)

[117] H. van Dam and M. Veltman, Nucl. Phys. B 22, 397 (1970)

[118] Y. Iwasaki, Phys. Rev. D 2, 2255 (1970)

[119] A. I. Vainshtein, Phys. Lett. B 39, 393 (1972)

[120] D. G. Boulware and S. Deser, Phys. Rev. D 6, 3368 (1972)

[121] A. I. Vainshtein, Surv. High Energy Phys. B 20, 5 (2006)

[122] V. A. Rubakov and P. G. Tinyakov, Phys. - Usp. 51, 759 (2008)

[123] C. de Rham, Liv. Rev. Relat. 17, 7 (2014)

[124] C. de Rham et al., arXiv:1606.08462v1 [astro-ph.CO]

[125] C. de Rham, G. Gabadadze and A.G. Tolley, Phys. Rev. Lett. 106, (2011)

[126] A. S. Goldhaber and M. M. Nieto, Phys. Rev. D 9, 1119 (1974)

[127] S. S. Gershtein, A. A. Logunov and M. A. Mestvirishvili, Doklady Physics 48, 282 (2003)

[128] S. S. Gershtein et al., Phys. At. Nucl. 67, 1596 (2004)

[129] S. S. Gershtein et al., Phys. Usp. 49, 1179 (2006)

[130] Yu. V. Chugreev, Phys. Part. Nucl. Lett. 13, 38 (2016)

[131] C. Talmadge et al., Phys. Rev. Lett. 61, 1159 (1998)

[132] C. Will, Phys. Rev. D 57, 2061 (1998)

[133] S. R. Choudhury et al., Astropart. Phys. 21, 559 (2004)

[134] L. S. Finn and P. J. Sutton, Phys. Rev. D 65, 044022 (2002)

[135] S. L. Larson and W. A. Hiscock, Phys. Rev. D 61, 104008 (2000)

[136] A. Cooray and N. Seto, Phys. Rev. D 69, 103502 (2004)

[137] C. Braxmaier et al. Exper. Astron. 34, 181 (2012)

[138] M. V. Sazhin, Sov. Astron. 22, 36 (1978)

[139] S. Detweiler, Astrophys. J. 234, 1100 (1979)

[140] D. Baskaran et al., Phys. Rev. D 78, 044018 (2008)

[141] D. Baskaran et al., Phys. Rev. D 78, 089901(E) (2008)

[142] K. Lee et al., Astrophys. J. 722, 1589 (2010)

[143] K. Lee, Class. Quant. Grav. 30, 224016 (2013)

[144] B. P. Abbott et al. (LIGO Scientific Collaboration and Virgo Collaboration), Phys. Rev. Lett. 116, 061102 (2016)

[145] B. P. Abbott et al. (LIGO Scientific Collaboration and Virgo Collaboration) LIGO Document P1500213-v27. arXiv:1602.03841

[146] B. P. Abbott et al. (LIGO Scientific Collaboration and Virgo Collaboration) 2016 Phys. Rev. X 6, 041015 (2016). LIGO-P1600088. arXiv:1606.04856

[147] M. Visser, Gen. Rel. Grav. 30, 1717 (1998)

[148] A. F. Zakharov et al., J. Cosmol. Astropart. Phys. JCAP05 (2016) 045

[149] A. F. Zakharov et al., EPJ Web of Conferences 125, 01011 (2016)

[150] N. Blind et al., arxiv:1503.07303 [astro-ph.IM]

[151] A. Ardeberg et al. 2014 An Expanded View of the Universe Science with the European Extremely Large Telescope, ed. M. Lyubenova and M. Kissler-Patig (ESO, Munich, 2014)

[152] W. Skidmore et al. Thirty Meter Telescope Detailed Science Case: 2015 (TMT Observatory Corporation, 2014) 